\title{
Aclaraciones Wittgensteinianas en torno al Color
}

\author{
Wittgensteinian Clarifications on Colour
}

ALEJANDRO TOMASINI BASSOLS

\section{Resumo}

En este trabajo argumento que sólo mediante un enfoque y un tratamiento wittgensteinianos se pueden disolver las pseudo-dificultades filosóficas concernientes a los colores (naturaleza, conocimiento, ubicación, etc.). Sostengo que, aunque los hechos que de los que se ocupa la ciencia son fundamentales para que pueda gestarse el lenguaje de los colores, lo que la ciencia (ya sea la física ya sea la fisiología) tiene que decir es enteramente irrelevante para inquietudes concernientes al significado, utilidad, enseñanza y aprendizaje, etc., del lenguaje de los colores. Asumiendo una perspectiva wittgensteiniana, se puede hacer ver que los problemas filosóficos concernientes a los colores son efectivamente artificiales, porque plantean interrogantes en los que los nombres de los colores no son usados. Lo que nos debe interesar es describir su aplicación, explicar para qué nos sirve el lenguaje de colores, qué utilidad de hecho presta dicho juego de lenguaje. Doy por sentado que las palabras para colores no se usan solas sino en oraciones, en conexión con verbos de percepción y recojo dos contrastes de verbos como 'ver'. Por una parte, 'ver' apunta tanto a propiedades objetivas de las cosas ('la botella es verde') como a experiencias visuales de los hablantes ('tengo la experiencia visual de algo verde'). Por otra parte, los verbos psicológicos como 'ver' están marcados por una asimetría fundamental entre la primera y la tercera personas. Ambas distinciones tienen consecuencias para el lenguaje de los colores, porque éste sirve para lidiar con el mundo a través de nuestras vivencias. Éstas, sin embargo, son

\footnotetext{
a Universidad Nacional Autónoma de México (UNAM), Cidade do México, México. Doutor, e-mail: altoba52@gmail.com
} 
relevantes en la medida en que quedaron lingüistizados, esto es, cuando los hablantes ya se volvieron usuarios de la terminología de los colores, es decir, ya adscriben y reconocen correctamente las propiedades cromáticas de los objetos. ¿Para qué nos sirve entonces el lenguaje de los colores? En primera persona ("Veo tu auto rojo desde aquí") indico que tengo ciertas vivencias que son gracias al lenguaje comprensibles para otros y le doy garantías a mi interlocutor de que puedo lidiar con objetos (reconocerlos, identificarlos, etc.); en tercera persona ("él trajo el balón del color que se le pidió") me permiten describir la conducta exitosa de alguien en procesos de reconocimiento y manipulación de objetos. La presuposición fundamental del enfoque wittgensteiniano (establecida en las Investigaciones Filosóficas y usada de ahí en adelante) es la existencia de una comunidad lingüística. Comprendemos entonces que los nombres para colores, como cualesquiera otras palabras, se aprenden a través de un proceso de enseñanza ostensiva, ensayo y error, interacción permanente entre hablantes y aprendiz, premios y castigos, etc. Aplico también la idea de que, contrariamente a como proceden los filósofos, las palabras para colores no se usan solas, sino en oraciones y en conexión con actividades, esto es, con formas de vida. Los conceptos de juegos de lenguaje y formas de vida son pues los instrumentos que se requieren para superar los enredos filosóficos en torno al color.

Palabras-chave: Wittgenstein. Lenguaje. Ver. Colores.

\section{Abstract}

In this paper I argue that it's only thanks to a Wittgensteinian approach and treatment that philosophical pseudo-difficulties concerning colour (nature, knowledge, location, etc.) can be dissolved. I hold that although the facts science is concerned with are indispensable for the language of colours to arise, what science (either physics of physiology) has to say is totally irrelevant for worries about the meaning, utility, teaching and learning, etc., of the language of colours. Assuming a Wittgensteinian perspective, it can be shown that philosophical problems concerning colours are indeed artificial, since philosophers raise questions in which the names for colours are not used. Accordingly, what should interest us is to describe their application, to explain what the language of colours is useful for, what the point of that particular language-game is. I take for granted that words for colours are not used alone but within sentences, in connection with perceptual verbs. I then point out two important contrasts of verbs like "see". On the one hand, "see" points both to objective properties of things ("the bottle is green") and to the speaker's visual experiences ("I have the visual experience of something green"). On the other hand, psychological verbs are marked off by a fundamental asymmetry between the first and the third persons. Both distinctions have consequences for the language of colours, for the latter helps us to deal with the objects of the world through our experiences. Our experiences, however, are relevant only insofar as they were previously "linguistisized", that is, once the speakers already became users of the terminology of colours, i.e., when they are able to ascribe and recognize the chromatic properties of objects properly. Now what is the language of colours useful for? In the first person ("I see your red car from 
here") I indicate to others that I have a certain experience which, thanks to our shared language, is understandable to them and I offer warrants that they can trust me in my dealings with objects (recognize them identify them, etc.); in the third person ("he brought the ball with the right colour"), they allow me to describe someone's successful behaviour in processes of identification and manipulation of objects. The fundamental presupposition of the Wittgensteinian approach (a result reached in the Philosophical Investigations and freely used from there onwards) is the existence of a linguistic community. Then we immediately understand that the names of colours, as any other word, are learnt through a process of ostensive teaching, trial and error, punishment and reward, a permanent interaction with speakers and so on. I also apply the idea that, contrary to the way philosophers tend to proceed, colour words are not used in isolation, but in sentences and in connection with activities, that is, forms of life. So, the concepts of language-games and forms of life are what is required to overcome philosophical puzzles concerning colour.

Keywords: Wittgenstein. Language. See. Colour.

\section{I) El problema}

Difícilmente podría negarse que, planteado en sus términos tradicionales, el tema filosófico del conocimiento y la naturaleza de los colores es un tema que sigue sin ser resuelto. Hay desde luego diversas teorías científicas al respecto, algunas de ellas tremendamente sofisticadas y precisas, pero es evidente que dichas teorías se fundan en una comprensión previa y por ende más fundamental de lo que son los colores y que es precisamente lo que sigue eludiéndonos. De ahí que podamos sostener a priori que las teorías científicas no puedan constituir una respuesta a la inquietud filosófica concerniente a los colores. En verdad, con el tema de la naturaleza de los colores confirmamos (una vez más) que el avance científico es pura y llanamente irrelevante para la resolución de las problemáticas filosóficas y de hecho es inclusive susceptible de complicarlas aún más. Esto se ve claramente cuando examinamos la confrontación entre los enfoques físicos y fisiológicos del color: en ambos casos se nos ofrecen explicaciones de orden causal sólidamente establecidas y de hecho imposibles de rechazar pero que, si no son debidamente contextualizadas, simplemente se excluyen mutuamente. De hecho, 
lo único que logran los grandes enfoques y tratamientos científicos del color es ilustrar a la perfección el dilema de elegir entre el punto de vista de acuerdo con el cual el color es una experiencia explicable en términos del sistema nervioso, de nuestros órganos perceptuales, etc., y el de que es más bien una propiedad abstracta pero objetiva de las cosas que pueblan el mundo. Lo que ni los científicos ni los filósofos de espíritu cientificista parecen comprender es que si bien ambas clases de teorías científicas pueden ser impecables para resolver diversas clases de problemas factuales, ninguna de ellas logra insertarse en el plano de la discusión filosófica, pues no pueden incidir en debates como el de la aclaración del significado de los términos para colores. Las teorías causales pueden ser, cada una en su ámbito, sumamente exitosas pero su éxito es ante todo un éxito práctico y no constituye ningún progreso concerniente a, por así decirlo, la esencia del color. Más bien, las teorías científicas presuponen nuestra conceptualización usual de los colores, la cual se deriva directamente de nuestras formas normales de hablar acerca de ellos y sobre esto último ni físicos ni fisiólogos tienen algo que decir. De hecho, el fundamento de los ulteriores desarrollos científicos concernientes a los colores está virtualmente contenido en nuestro uso común del vocabulario de los colores. Es gracias a que tanto físicos como fisiólogos saben lo que son los colores, esto es, que conocen los significados de los nombres de los colores que posteriormente pueden realizar sus respectivos experimentos y desarrollar sus nuevos conceptos de color, i.e., los conceptos físico y fisiológico del color, respectivamente, construidos ambos en conexión con sus respectivas formas de vida, esto es, con sus respectivas clases de experimentos, instrumentos, léxicos, etc. Así, cuando decimos, e.g., 'la mesa es roja', por una parte le adscribimos al objeto "mesa" una propiedad particular, una cualidad que el objeto tiene, así como tiene un cierto peso o una determinada forma. La óptica desarrolla esta faceta del concepto. Y por la otra damos a entender que tuvimos una determinada experiencia, a saber, la experiencia de lo rojo De esta segunda faceta se encarga la fisiología. Tanto el físico como el fisiólogo saben qué investigar y cómo hacerlo precisamente porque ya manejan el concepto de color y porque ya saben lo que es "lo rojo". Claramente entonces, los usos científicos de "lo rojo" o de "ver rojo" son desarrollos conceptuales que tienen un punto de partida común, a saber, el uso común de la palabra 'rojo'. 
Así, pues, el vocabulario de los colores se usa simultáneamente en conexión con objetos y en conexión con experiencias personales, con estados de percepción. Ahora bien, salvo cuando lo que se quiere es ofrecer definiciones ostensivas, en general los nombres de los colores no se usan solos sino en oraciones y en especial con verbos como 'ver'. De ahí que 'ver' sea, por así decirlo, un verbo de dos dimensiones: alude simultáneamente a propiedades de objetos y a las experiencias visuales del sujeto percipiente: por una parte, el verbo 'ver' tiene en general un complemento de objeto directo, es decir, siempre que se ve se ve algo y ese algo normalmente es un objeto que tiene determinadas cualidades perceptuales, pero por la otra 'ver' es también un verbo psicológico y en este otro sentido alude a una vivencia particular, a lo que los filósofos llaman un ‘estado interno’. ¿No es esto curioso? Debería ser obvio que esta doble vía de significación no es ni arbitraria ni gratuita; si el lenguaje la traza no es por casualidad. Lo que sucede es que, en condiciones normales, nosotros efectivamente percibimos las propiedades que los objetos de hecho tienen, pero es perfectamente posible que un objeto tenga propiedades cromáticas que no percibimos o que tengamos una experiencia visual que no corresponde a ninguna propiedad de un objeto. Ambas posibilidades están contempladas en nuestro lenguaje de percepción y de adscripción de colores. Ninguna de esas posibilidades, sin embargo, hace que el lenguaje de los colores pierda su objetividad. Como argumentaré más abajo, es gracias a la naturaleza compartida o social del lenguaje de los colores que la experiencia del color es algo que se puede compartir, debatir, cuestionar, etc., y que las adscripciones de colores no son caóticas, arbitrarias o meramente subjetivas. Es por ello que podemos decir que aunque cada quien tiene su propia vivencia de todos modos todos vemos lo mismo. Huelga decir que el funcionamiento normal del lenguaje presupone que tanto el mundo físico como nuestro aparato perceptual son estables, pero este reconocimiento no implica que sea entonces tarea de la ciencia explicar la naturaleza del color.

Como era de esperarse, las dos (por así llamarlas) 'dimensiones semánticas’ incorporadas en el uso del verbo 'ver', así como en el de otros verbos de percepción y en el vocabulario de los colores, dan lugar de manera natural a diferentes clases de preguntas, 
esto es, a preguntas que son significativas en un caso pero no en el otro, y a la inversa. Por ejemplo, si mi interés en 'ver' está en su uso como verbo que tiene un complemento

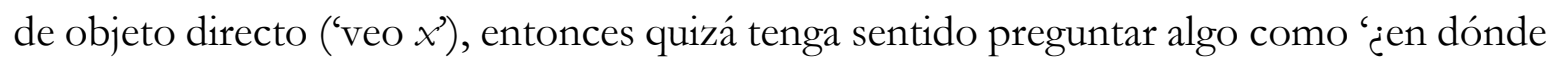
está el color?', en tanto que si me concentro en el uso de 'ver' como verbo de experiencia preguntar por la ubicación del color no tiene el menor sentido. Preguntas como ‘ ¿Dónde en mi experiencia del color?' son simplemente absurdas. Tiene sentido preguntar por la ubicación del color, porque al plantearla se pregunta por una propiedad de un objeto y entonces ciertamente podemos hablar de ubicación o de forma, pero nada de eso vale para 'ver' en su segunda clase de aplicación. No reconocer estas diferencias de uso automáticamente conduce a que se plantee toda clase de preguntas ilícitas y a que se les dé por buenas, esto es, por legítimas, cuando en realidad sin simplemente absurdas. Por mi parte, deseo sostener que el paso crucial para la superación del problema filosófico de la naturaleza del color consiste en entender que la objetivización del color requiere forzosamente de un proceso de lingüistización de las experiencias visuales. En el nivel de formación conceptual básica, las explicaciones causales que posteriormente se ofrecen, sean físicas o fisiológicas, son irrelevantes. Las explicaciones científicas vienen a cuento sólo si de lo que se habla es de creación de nuevos significados. Es obvio que en el plano del lenguaje común los juicios en los que empleamos palabras o nombres de colores no se ratifican o rechazan por lo que la física y la fisiología determinen. Lo más que se podría sostener sería que si los objetos del mundo físico y nuestro aparato perceptual carecieran de la estabilidad que sabemos que tienen, entonces nuestros juegos de lenguaje de percepción y de colores serían drásticamente diferentes, puesto que nuestra experiencia visual sería diferente de un modo que nos resulta aquí y ahora inimaginable e imposible de describir. Ahora bien, si efectivamente nuestra terminología y nuestros juicios en los que aparecen palabras para colores no adquieren los sentidos que tienen por los hechos subyacentes, esto es, físicos o fisiológicos, entonces ¿en que se fundan? Mi punto de vista es que el juego de lenguaje de los colores se estructura como lo hace en virtud de que reaccionamos de igual modo frente a las estimulaciones visuales y por las actividades que efectuamos en conexión con los objetos de los que hablamos. En otras palabras, lo que tenemos que rescatar es la utilidad que prestan los nombres de los colores, el uso que 
hacemos del lenguaje de los colores. Como intentaré hacer ver, es en ese aparentemente inocuo cambio de óptica que en gran medida radica la novedad y la potencia del enfoque wittgensteiniano.

\section{II) Enredos tradicionales en torno al color}

Es evidente de entrada que los colores, como cualquier otro tema, plantean retos intelectuales interesantes, retos de carácter empírico que se van respondiendo paulatinamente. De hecho, la ciencia del color está tan avanzada que casi podría decirse que desde un punto de vista científico el tema del color es un tema agotado. De hecho, no hay mucho más que decir. En contraste con ello, sin embargo, hay preguntas y planteamientos en relación con los cuales no se ha realizado absolutamente ningún progreso. Es el caso de los interrogantes filosóficos concernientes al color. ¿Cómo nos explicamos semejante fenómeno? Para responder, quizá lo primero que deberíamos hacer sería presentar una breve lista de preguntas que los filósofos plantean a fin de tener una idea de qué clase de inquietudes estamos hablando. Preguntas filosóficas típicas son:

a) ¿qué es el color?

b) ¿en dónde está el color?

c) ¿cómo podemos saber si mi experiencia de, e.g., lo rojo coincide con la experiencia que otros dicen tener de lo rojo?

d) ¿cómo podemos saber si 'rojo' tiene un único significado o si no significa algo diferente para cada quien?

e) ¿'es lo rojo una propiedad de los objetos (cualidad primaria) o una propiedad de la mente percipiente (cualidad secundaria)? 
No creo que pueda sostenerse que el resultado en el que desembocan las disputas filosóficas en torno al color, a saber, el escepticismo filosófico, sea un resultado deliberadamente buscado por los filósofos, pero que es prácticamente inevitable caer en él es algo que se comprende tan pronto detectamos los rasgos característicos de las preguntas que ellos formulan. A mi modo de ver, las preguntas filosóficas referentes al color combinan dos propiedades: por una parte, son preguntas ingenuas, lo cual se explica por el hecho de que están planteadas tomando como modelo esquemas gramaticales sumamente simples (preguntas de la forma ‘qué es x?’) y, segundo, son preguntas en las que se inquiere por el significado de palabras consideradas en forma aislada, con lo cual automáticamente se vuelve imposible entender en qué consiste la contribución semántica de las palabras al sentido de lo que se dice (en general, expresado mediante oraciones). Vicios argumentativos como estos llevan al socorrido juego filosófico estándar de los "mundos posibles" y hacen que las discusiones filosóficas a menudo desemboquen en ideas como la de que es lógicamente posible que todos coincidamos en juicios pero que, no obstante, difiramos en experiencias. Eso es un resultado escéptico, puesto que se sigue que el hablante normal nunca sabe qué son los colores ni si otros saben lo mismo que él cuando hablan de los colores de las cosas. Ello es un claro indicio de que los planteamientos tradicionales emergen de graves confusiones conceptuales.

El esquema de los enfoques filosóficos, independientemente de las posiciones que se puedan desarrollar, es básicamente el mismo: sobre la base de preguntas desorientadoras, esto es, de preguntas que en realidad no apuntan a ningún problema específico así como están formuladas, se plantean pseudo-problemas para los cuales sólo puede haber como respuesta teorías absurdas las cuales, dicho sea de paso, proliferan. Ahora bien, no obstante lo florido del jardín de los enredos filosóficos, en general los vicios discursivos y argumentativos en los que incurren los filósofos son básicamente los mismos: se pregunta por el significado de los términos para colores en forma aislada (e.g., ¿cuál es el significado de 'amarillo’?), se presupone siempre alguna teoría del significado (en general, alguna teoría de corte crudamente referencialista de los nombres) y se pretende explicar el significado en términos de objetos de alguna índole (físicos, mentales, 
fenomenológicos, intencionales, materiales, trascendentales, emergentistas y así ad libitum). En este sentido, la verdad es que estamos básicamente en la misma situación que en la que se estaba en tiempos de Locke: los colores no pueden ser otra cosa que "propiedades secundarias", las cuales se diferencian de las primarias (forma, número, peso, etc.) porque, de alguna manera, son "puestas" en los objetos por la mente humana. Aparentemente, la "prueba" de que ello es así la tenemos en el hecho de que si pasamos a un cuarto oscuro el objeto que estemos examinando éste continuará teniendo sus propiedades primarias, pero habrá perdido por completo sus cualidades secundarias y muy en especial sus colores. El argumento, sin embargo, es notoriamente falaz. Es perfectamente imaginable que pasemos un objeto sólido a un cuarto con una temperatura que hace que se desintegre pero que la sustancia que quede siga teniendo el mismo color que el objeto original. ¿Habría entonces que inferir que propiedades como la forma o el peso del objeto, vistas en general como propiedades primarias, no son propiedades reales del mismo? Sería absurdo razonar de esa manera. Lo mismo en el caso del color. Lo que habría que decir es no que el objeto pierde su color cuando lo paso a un cuarto oscuro sino que, por falta de luz, yo ya no puedo percibirlo. Pero no hay ningún misterio en ello: nuestros conceptos de colores se formaran en condiciones normales y lo que en casos así se pretende es que sigan funcionando sólo que en condiciones enteramente anómalas. Después de todo, el que se modifiquen las condiciones normales de aplicación de conceptos tiene que tener consecuencias de alguna índole al momento de volver a aplicarlos. ¿O no?

\section{III) El enfoque wittgensteiniano}

Tal vez no esté de más recordar que el tratamiento de los colores por parte de Wittgenstein pasó por tres grandes fases, a las que podemos referirnos como las fases 'lógica', 'gramatical' y 'fenomenológica'. La fase lógica, esto es, la del Tractatus, termina en un rotundo fracaso puesto que Wittgenstein se ve forzado o a renunciar a todo intento 
de aclaración del sentido de proposiciones como 'esto es rojo' (que epistemológicamente es una proposición última, irreductible, simple, etc.) o a abandonar su concepción de las proposiciones elementales como lógicamente independientes entre sí. Enfrentado a este dilema, lo más que Wittgenstein llega a sostener es que tenemos que conocer mejor la estructura lógica del color para poder aclarar por qué 'a es rojo' es una proposición elemental del lenguaje natural. Como es bien sabido, no hay tal conocimiento por lo que la propuesta es totalmente fallida. Su segunda fase arranca justamente con el propósito de dar cuenta de ese problema precisamente, es decir, de explicar cómo se relacionan entre sí las proposiciones que desde el punto de vista del conocimiento empírico son las fundamentales, las más básicas, como por ejemplo 'a es verde'. Desde su primer acercamiento en "Algunas Observaciones sobre la Forma Lógica" Wittgenstein se va orientando en una dirección opuesta a su atomismo lógico radical, pues él va poco a poco descubriendo que las proposiciones funcionan en grupos, se apoyan mutuamente, forman sistemas. Este nuevo enfoque lo lleva a interesarse no ya por la lógica del lenguaje sino por su "gramática" y a efectuar entonces análisis gramaticales de diversas nociones. Su trabajo culmina con el ahora célebre octaedro de los colores, una forma gráfica de exponer la gramática del vocabulario de los colores, esto es, entre otras cosas las conexiones que fijan el ámbito de lo significativo cuando lo que se usa son palabras (nombres) para colores. Y, por último, en una tercera fase, hacia el final de su vida, Wittgenstein enfrenta problemas que los colores plantean pero que ya no son de carácter lingüístico, sino estrictamente fenomenológicos. Esta labor, recogida en lo que fue publicado como 'Observaciones sobre los Colores', es un análisis de la experiencia del color. Sobre esta última etapa diré posteriormente algunas palabras.

En este trabajo no me propongo realizar una labor de exégesis de textos wittgensteinianos. Tal vez sí deba advertir, sin embargo, que presupongo ciertos resultados generales establecidos hace ya muchos años por Wittgenstein. Por ejemplo, "presupongo" una comunidad lingüística. Nótese que tan sólo esta "presuposición” basta para que el tema del color cambie radicalmente de cariz. Desde esta perspectiva nosotros planteamos no preguntas banales, descontextualizadas y desorientadoras como ‘qué es el color?’ o ‘qué significa “violeta”?’, esto es, preguntas que son significativas sólo si se 
asume que ya se sabe lo que son los colores y que son por lo tanto redundantes. Para nosotros, en cambio, es de suma importancia en primer lugar la cuestión de cómo los aspirantes a hablantes logran convertirse en usuarios del juego de lenguaje de los colores. Esto es crucial puesto que es evidente que los nombres de los colores son enseñables y aprendibles y, por lo tanto, compartibles. A diferencia de lo que pasa con los enfoques centrados en la primera persona, el mero planteamiento wittgensteiniano deja en claro que no podría darse el caso de que sólo yo (i.e., sólo cada quien) supiera qué y cómo significan las palabras para colores y que los demás no, puesto que para que ello fuera posible yo habría tenido que entrenarme a mí mismo en el uso del vocabulario de colores, es decir, tendría yo que haber interiorizado por cuenta propia las reglas de uso de 'rojo', 'más oscuro que', etc., y eso es pura y llanamente imposible (por no decir 'ininteligible'). Recordemos, de paso, que creer que se siguen reglas no es seguir reglas. Nadie, por consiguiente, se puede instruir a sí mismo en el uso de lenguaje, por la simple razón de que el lenguaje ni es innato ni es (en un sentido radical) privado, sino que es un instrumento social, público y compartido. El enfoque wittgensteiniano es, naturalmente, el de los juegos de lenguaje y las formas de vida y dicho enfoque de inmediato exhibe el carácter declaradamente artificial de las preguntas filosóficas, pero deja en claro además que todo intento de aclaración filosófica concerniente a los colores tiene que girar en torno a las aplicaciones o usos que se hagan de los nombres de los colores. Por ello, todo esfuerzo de esclarecimiento concerniente a nuestros conceptos de colores tiene que realzar su utilidad, su practicalidad. Espero dejar en claro esto en lo que sigue.

\section{IV) Tratamiento wittgensteiniano del tema de los colores}

Es incuestionable que el cambio de óptica que con Wittgenstein se operó es en verdad de primera importancia, entre otras razones porque se dejaron atrás los enfoques que podríamos llamar 'sustancialistas' del significado y ello en favor de lo que en otros escritos he llamado el 'enfoque praxiológico'. Esto quiere decir, inter alia, que no son los 
objetos mismos los que dotan de significado a una palabra, sino que éste surge más bien con las actividades que los hablantes realizan en conexión con el uso de la palabra relevante, en nuestro caso con los nombres de los colores. De hecho, me parece que pretender hablar de los colores, como normalmente se hace en filosofía, como si fueran “objetos" es una forma estrafalaria o más bien abiertamente descabellada, y ciertamente ilegítima, de hablar. Los colores no son objetos, como no lo son las nubes o las sombras, puesto que no predicamos de ellos lo mismo que predicamos de los objetos espaciotemporales. No tiene mayor caso, por otra parte, hacer en abstracto preguntas de la forma ‘¿qué significa “rojo”?’, porque en el lenguaje natural no empleamos palabras sueltas, sino en oraciones. Un nombre no es más que una etiqueta para posteriormente decir algo acerca de algo, es decir, es un movimiento preparativo para poder decir algo, pero obviamente el mero hecho de usar un nombre no permite adivinar su significado. Lo que nosotros entendemos es el movimiento en el juego de lenguaje. Se sigue que el significado de las palabras componentes es algo que sólo se puede determinar después de haber dicho algo, no antes, y para ello se requieren oraciones. Por ello es fundamental describir lo que se hace cuando empleamos oraciones en las que aparecen nombres de colores. Así, quien usa nombres de colores los usa en oraciones y si usa oraciones ya sabe qué hacer con ellas. $\mathrm{Y}$ ¿qué es lo que hacemos cuando hablamos de los objetos en términos de sus colores? Los colores permiten identificar objetos, clasificarlos, ordenarlos, describirlos, contarlos, etc.

Es, pues, en conexión con las formas de vida, es decir, con las actividades colectivas en relación con las cuales se emplearon palabras como 'rojo', 'amarillo’ y demás, que podemos determinar su significado. Después de todo, la razón de ser última del lenguaje de los colores tiene que ser de carácter práctico, no meramente especulativo, contemplativo o decorativo. Es obvio además que preguntas como ‘¿qué es lo rojo?’ o ‘¿qué significa 'verde'?' sólo se entienden en la medida en que ya se sabe lo que son los colores, es decir, en la medida en que ya interiorizó el concepto de color, porque si no fuera así ¿cómo podría alguien responder a semejantes preguntas? Alternativamente: ¿cómo puede alguien responder a la pregunta ‘¿qué es el tres’? si no tiene la idea de número? Una vez más, queda en claro que las preguntas filosóficas usuales en realidad son a menudo 
grandes peticiones de principio. Los filósofos ya saben lo que son los colores, pero plantean preguntas al respecto como si no lo supieran. Algo, por lo tanto, debe estar mal en sus planteamientos. Por otra parte, es importante no olvidar que el juego de lenguaje de los colores está integrado en o forma parte de juegos de lenguaje más amplios, a saber, el juego de lenguaje de los objetos materiales y el juego de lenguaje de la percepción visual. Como dije, el lenguaje de los colores sirve para distinguir entre objetos y más concretamente entre objetos que vemos. Salvo para propósitos muy específicos, nadie habla de los colores al margen ya sea de objetos ya sea de experiencias. Es obvio que siempre que vemos vemos algo y que lo que vemos tiene que tener algún color. Aunque el verbo 'ver' se puede emplear sin hacer referencia explícita a colores ('veo un perro corriendo'), es obvio que no podemos simplemente tener la experiencia de ver sin que aquello que veamos tenga algún color. No tiene el menor sentido hablar de "ver" a secas o en abstracto, sino que siempre que vemos, vemos algo y ese algo es coloreado. El verbo 'ver', como ya se dijo, cumple una doble función: alude a una experiencia, pero también a objetos y sus propiedades y esta doble función está siempre presupuesta en nuestro discurso sobre colores. En la experiencia visual distinguimos objetos no sólo por sus dimensiones, pesos, formas, etc., sino también por sus colores, porque el mundo que percibimos nosotros, los humanos, es coloreado. Es, pues, comprensible que para los sentidos de nuestras expresiones en las que empleamos conceptos de colores aquello para lo que sirven, lo que nos permiten hacer, sea lo que es relevante. Nosotros extraemos el sentido de las palabras de la clase de utilidad que prestan al ser empleadas, no de sus condiciones empíricas de existencia, descubiertas siempre posteriormente a la asimilación conceptual.

Los conceptos de colores se usan en coordinación con descripciones de objetos, pero en la medida en que se usan también en relación con estados psicológicos sucede con ellos lo mismo que con los conceptos psicológicos en general: presentan una asimetría en su uso entre la primera y la tercera personas. Aunque es obvio que no sólo se puede distinguir entre objetos por sus colores, lo que es evidente es que si alguien distingue objetos por sus colores entonces ve, sabe lo que es ver y, en algún sentido, sabe 
lo que son los colores. Cuando se usan verbos de percepción en primera persona dando lugar a oraciones en las que aparecen nombres de colores lo que el hablante hace es informar, indicarle o darle a conocer a los demás que está en posición de describir, clasificar, ordenar, etc., objetos e implícitamente da a entender que tiene una experiencia visual particular sólo que lo que dice no versa sobre ésta. Podría decirse que se le da por supuesto, que se le asume, presupone, etc., pero en todo caso eso que le pasa al sujeto no entra en la determinación del sentido de lo que expresó, en lo que él le dice a otros. Por otra parte, si se afirma de alguien que ve, lo único que no se puede estar queriendo decir es que la persona en cuestión tiene lo mismo que yo cuando digo de mí mismo que veo, porque eso sería tratar de reducir el uso en tercera persona al uso en primera persona y eso claramente es, por razones harto conocidas, una maniobra que no tiene el menor viso de éxito. Pero entonces ¿qué quiero decir cuando afirmo de alguien "que ve"? La respuesta es evidente de suyo: yo me percato de que la otra persona puede realizar todas las actividades que yo puedo realizar gracias precisamente a que puedo tener eso que llamamos 'experiencias visuales': es porque la otra persona no se tropieza, que va directamente a los objetos que le pido que me traiga, que trae el que le pido y no otro, porque no se golpea, porque no va a tientas, etc., que decimos de la persona que ve. Es para eso, en relación con eso que se construyó el concepto de ver. En tercera persona, por lo tanto, 'ver' no es usado como un verbo de experiencia, sino como uno eminentemente conductual. De ahí que, de hecho, las adscripciones del verbo 'ver' sean ante todo adscripciones de capacidades físicas, corporales, de movimiento, de coordinación, etc. En resumen: cuando afirmo que veo lo que quiero decir es que quiero darle garantías a mi interlocutor de que, en conexión con algo que me pasa, puedo realizar exitosamente todas las actividades que normalmente realizan las personas que ven. Naturalmente, todos los hablantes espontáneamente asumimos que a todas las personas que dicen 'yo veo' algo les pasa, pero el punto es que su, por así decirlo, "experiencia bruta" no entra en el significado de lo que se afirma, no forma parte de él. Es sólo cuando esa experiencia quedó lingüistizada que entonces se transmite o comunica a otros de modo que los demás entienden lo que se quiere decir, en este caso, darles garantías de que uno puede desplazarse, distinguir entre objetos, clasificarlos, etc., sin problemas, exitosamente. Y decir de alguien que ve 
es afirmar que esa persona puede moverse exitosamente en un mundo de objetos. Sin duda alguna y como ya se dijo, el juego de lenguaje de ver y de las adscripciones de colores presupone como cuestión de hecho la estabilidad del mundo, si bien ésta es irrelevante para la significación de las palabras.

Me parece que podemos sintetizar la situación como sigue: nosotros, los usuarios del lenguaje natural, reaccionamos de determinada manera frente a los estímulos externos, tanto por las propiedades físicas de los objetos con los que nos topamos como por el funcionamiento de nuestro aparato perceptual. Dado que los seres de nuestra especie reaccionamos del mismo modo, hacemos más o menos las mismas muecas, emitimos los mismos gritos, etc. (piénsese, por ejemplo, en nuestras reacciones frente al fuego: ¿hay algún ser humano normal que reaccione con una sonrisa frente a una quemadura de tercer grado? Lo mismo pasa con nuestras reacciones en conexión con nuestras percepciones visuales). Ahora bien, es sobre la base de que reaccionamos de la misma manera que se nos puede enseñar a identificar colores introduciendo sus nombres. En general, este procedimiento funciona: aprendemos que ese color se llama 'amarillo', aquel otro 'negro', etc., porque todos reaccionamos del mismo modo frente a las emisiones de los sonidos, gestos, indicaciones, tonos de voz, etc., así como reaccionamos del mismo modo frente al dolor o el placer (rehuimos el primero, buscamos el segundo) y aprendemos a distinguirlos verbalmente. Obviamente, hay una reacción espontánea frente al dolor que se da en el caso de los colores, porque no hay tal cosa como reacción frente a lo rojo, reacción frente a lo azul, etc. Pero en ambos casos es sobre la base de una fundamental concordancia en reacciones que se puede construir y hacer operativo el lenguaje de las sensaciones y el lenguaje de la percepción de colores.

¿Cómo se aprende entonces a emplear el vocabulario de los colores o, si se prefiere, cómo puede alguien enseñarle a otro a identificar colores, esto es, a usar correctamente los nombres de los colores? Dicho de otro modo: ¿cómo es que nos volvemos usuarios de dicho juego de lenguaje? Lo primero que hay que señalar es que para cuando el niño empieza a usar los nombres de los colores ya pasó por un cierto 
periodo de entrenamiento verbal y de coordinación de sus sentidos, en particular entre los datos del tacto y los de la visión. El niño aprende a estirar la mano y a tocar objetos que poco a poco va identificando verbalmente. En este proceso es permanentemente alentado por los usuarios normales del lenguaje que lo rodean. Si el niño toca la pelota roja cuando le dicen 'la roja', entonces se le aplaude, se le mima, se le da un dulce, etc., es decir, se le premia, se le estimula, y si toca otra entonces, suavemente, se le reprime: se le habla en un determinado tono, no se le da lo que toca, etc. Obviamente, se cometen errores, pero si el aprendiz es normal éstos son cada vez más esporádicos hasta que simplemente deja de cometerlos. Decimos entonces que ya entendió el significado de la palabra, e.g., 'verde'. La interiorización del lenguaje de los colores, por lo tanto, resulta de una cierta coordinación entre repetidas aplicaciones de palabras, definiciones ostensivas, y reacciones naturales sometidas a procesos de estimulación, premios si son acertadas, castigos si son erradas. La situación alarmante es obviamente aquella en la que el niño resulta incapaz de actuar como los demás. Si la coordinación entre tacto y movimientos resulta infructuosa y si el aprendiz no logra rebasar el plano de la enseñanza ostensiva, entonces decimos que el niño "no ve" y lo que queremos decir es precisamente que él no puede realizar las acciones que la vista permite realizar. Automáticamente pensamos que al niño "algo le pasa", que no tiene la experiencia que llamamos 'ver'. Lo que entonces hacemos es buscar las causas de su no visión, esto es, de su ceguera, puesto que sólo en condiciones muy especiales nos inclinaríamos a pensar que si no ve es porque la realidad fuera de él se modificó! Lo que se pasa a examinar entonces es su equipo fisiológico, porque lo que asumimos es que él no está capacitado para interiorizar el juego de lenguaje de los colores o, más en general, de la visión. Nadie niega que el lenguaje de la percepción, visual u otra, sólo pueda gestarse si el cerebro, el sistema nervioso, etc., funcionan normalmente, pero de ello no se sigue que la percepción de colores sea lo mismo que el funcionamiento normal de la corteza cerebral, del sistema nervioso y demás. Ciertamente, el lenguaje de los colores depende existencial u ontológicamente del equipo fisiológico y neurofisiológico del sujeto, pero no es de éste directamente que brota el significado de las palabras de colores. Por eso buscar el color en el cerebro es lo más absurdo que se le podría haber imaginado a alguien. El significado de las palabras para colores obviamente 
depende existencialmente tanto de nuestras capacidades perceptuales como de las estructuras físicas de los objetos, pero él más bien se estructura y opera en función de las actividades que los humanos realizamos en conexión con los objetos del mundo. Por supuesto que no todas las actividades de los hablantes tienen que ver con la percepción visual y, por ende, con los colores. Un ciego puede distinguir entre objetos por sus formas, texturas, olores o pesos, pero no por sus colores, pero eso lo sabemos porque él no tiene la capacidad de moverse con fluidez en el mundo de los objetos de quienes sí ven. Carecer de esa peculiar capacidad de interactuar con los objetos que tiene la gente “normal” es lo que llamamos ‘ser ciego' o 'no ver'.

\section{V) El conocimiento del color}

Si el tema de los conceptos de colores empleados en tercera persona conduce directamente a las actividades humanas, considerado desde la perspectiva de la primera persona a lo que nos lleva es más bien a cuestiones de fenomenología. Es obvio que el tratamiento de cualquier problema fenomenológico presupone que ya se asimiló el juego de lenguaje de la percepción visual, el cual incluye al de los colores, y se "asume" por consiguiente que ya se captaron las reglas de aplicación de los nombres de los colores. En esta etapa no es la elucidación del significado, el análisis gramatical lo que nos interesa, sino la descripción de la experiencia del color. No nos interesa analizar 'veo rojo' o 'esto se llama "rojo", sino lo que pasa (por dar un ejemplo) con lo rojo, cómo se comporta lo rojo en nuestro campo visual, cómo se comporta frente al blanco, frente a la luz, etc. Dicha descripción será verdadera o falsa, acertada o desacertada, exacta o imprecisa, etc., pero será objetiva en el sentido de que cualquier otro hablante/percipiente podrá ratificar o rechazar las descripciones que se ofrezcan, puesto que a estas alturas estamos ya asumiendo que los conceptos de los colores son compartidos y que no hay ambigüedades al respecto. 
Es en sus notas publicadas bajo el título de Observaciones sobre los Colores que Wittgenstein realiza un formidable trabajo de análisis de la experiencia del color. Lo que tiene que decir es aparte de esclarecedor original en grado sumo. Si para efectuar análisis gramaticales se requiere estar muy atento a los giros lingüísticos, a las aplicaciones de las palabras, a los contextos discursivos, etc., para efectuar análisis fenomenológicos se requiere ser un extraordinario observador. Es asombrosa la cantidad de observaciones que Wittgenstein nos regala en unas cuantas páginas. Por ejemplo, su análisis del color blanco y de las relaciones de éste con otros colores es impresionante: el blanco hace rebotar la luz, no la deja pasar, hace que otros colores se diluyan, es el color más claro, el negro absorbe la luz, etc. Los problemas de la fenomenología del color son apasionantes, pero no siendo éstos nuestros temas no nos adentraremos aquí en lo que es la última fase de la reflexión de Wittgenstein sobre los colores.

Ya argumenté en favor de la idea de que la justificación última del juego de lenguaje de los colores radica en su practicalidad. No lo tenemos ni como ornato ni por gusto, sino porque dada nuestra estructura cognitiva y la naturaleza de la realidad, dicho juego de lenguaje es indispensable e insustituible. Los colores son importantes para nosotros no porque nos alegran la vista, sino por lo que nos permiten hacer, a saber, lidiar con los objetos del mundo. El juego de lenguaje de los colores, en efecto, es un mecanismo praxiológico (o sea, no meramente "lingüístico") para la descripción y manipulación de los objetos, un mecanismo lingüístico que si bien depende existencialmente de nuestro aparato perceptual, concretamente del visual, y de la estructura de la realidad, funciona en conexión con nuestras actividades. Así como les adscribimos dimensiones, tamaños, pesos, distancias, etc., a los objetos, así también les adscribimos colores. El juego de lenguaje de los colores es una forma alternativa que tenemos de describirlos y de manipularlos. Hablar acerca de los colores es simplemente hablar de una forma peculiar de distinguir entre objetos. Ahora bien, descripciones así son posibles porque, como ya dije nosotros coincidimos en reacciones, porque reaccionamos de la misma manera frente a las emisiones de palabras y frente a las estimulaciones visuales. Es sobre la base de esa fundamental concordancia en reacciones que se vuelven posibles las adscripciones de colores. Distinguimos entre objetos rojos y 
objetos verdes porque cuando empezamos a hablar reaccionamos como nuestros maestros esperan que lo hagamos y no de otra manera. $\mathrm{Y}$ es así que paulatinamente incorporamos el vocabulario de los colores. Podemos entonces sin problemas coincidir en nuestros juicios dado que para entonces compartimos ya una misma plataforma lingüística: aprendimos a reaccionar ante la emisión de las palabras de los modos apropiados y usarlas al modo como los demás las usan. Por eso la supuestamente imaginable situación de que usemos todos de manera consistente una misma palabra, digamos 'verde', pero que tengamos experiencias distintas, es mera mitología filosófica. Tener la experiencia de lo rojo no es nada más tener una experiencia, por así llamarla, 'orgánica', sino tener la experiencia de una propiedad de un objeto a la que llamamos 'roja'. Así, pues, saber qué es lo rojo es equivalente a emplear, a estar capacitado para usar la palabra 'rojo' de la manera que socialmente se considera como apropiada, correcta, congruente. Generalizando: saber lo que son los colores no es nada más "tener ciertas experiencias": es estar capacitado para hacer pasar sus experiencias visuales meramente orgánicas por el tamiz del lenguaje, que es una institución social, esto es, tener ciertas experiencias siendo un usuario del juego de lenguaje de los colores. Si alguien responde correctamente a órdenes como 'señala el objeto guinda', 'recoge el lápiz morado', 'patea la pelota lila', 'escribe en el cuaderno violeta', 'ponte el suéter de color índigo' y así sucesivamente, ese alguien sabe lo que son los colores. No hay un conocimiento más profundo de ellos. O sea, saber cómo se llaman, poder identificar y re-identificar colores, distinguir un color de otro, no equivocarse en la aplicación de los nombres de colores, etc., eso es saber qué son los colores. No hay nada más que añadir. Todo lo que se requiere es disponer de la técnica del lenguaje de los colores, para que pueda decirse que el usuario "sabe" lo que son los colores, que "conoce" la "naturaleza última" de los colores. Obviamente, saber qué son los colores no es lo mismo que estar al tanto de lo que afirman las últimas teorías científicas acerca de ellos. Eso claramente es otro asunto y confundir ambos planos es cometer graves errores categoriales.

Un último punto que quisiera mencionar es el siguiente: parecería que hay hablantes que, de alguna manera, "saben” más sobre los colores que otras personas, así 
como al parecer hay también personas que logran distinguir más matices que otras. El caso ya muy gastado de los esquimales viene a cuento: parecería que ellos tienen más conceptos de blanco que gente que vive en otras latitudes. Todo ello llama la atención, pero no tiene nada de misterioso. Los sentidos son algo que se educa y se desarrolla. Hay gente que, por algún entrenamiento especial y de carácter eminentemente inductivo, aprende a combinar colores (pinturas, por ejemplo) de un modo como usuarios estándar del lenguaje de los colores no sabrían cómo hacerlo. Pero esto es un asunto factual, de desarrollo perceptual, no conceptual. No todos los hablantes tenemos el mismo vocabulario y, por lo tanto, no todos tenemos las mismas posibilidades de descripción unos que otros, pero esas diferencias son en última instancia de carácter empírico. Lo que es importante entender es que todos asumimos que reaccionamos más o menos de la misma forma frente a los mismos estímulos: frente al agua fría, frente al fuego, frente a lo duro, etc., y frente a los colores. Y así como hay gente más resistente al dolor que otras, así también hay personas que disciernen mejor o más finamente que otras entre colores, que perciben matices que otros no perciben, qué han aprendido mejor que otros a combinar colores, etc. Es obvio, sin embargo, que todas esas diferencias emergen de un mismo aparato conceptual básico compartido. Una vez que nos volvimos usuarios de los conceptos de colores el manejo de los colores, de pinturas de autos por ejemplo, se vuelve un asunto de habilidades personales. Los juegos de lenguaje de los colores, como cualquier otro, pueden expandirse y aunque no necesariamente todos los hablantes son automáticamente usuarios de sus desarrollos, ramificaciones o extensiones, potencialmente sí lo son. Ello dependerá de su participación en las correspondientes formas de vida. Naturalmente, en la medida en que las nuevas prácticas lingüísticas están asociadas con objetos, instrumentos, actividades, etc., que no están al alcance de los hablantes normales éstos no estarán en posición de incorporar los nuevos juegos de lenguaje. Por estas y otras razones no podemos dejar de enfatizar la importancia y el carácter decisivo de la noción de forma de vida y de su utilidad para dar cuenta de enredos filosóficos. El caso de los colores es, creo, un buen ejemplo de ello.

\section{VI) Consideraciones finales}


Yo creo que, para redondear lo que sería nuestro punto de vista, habría que decir lo siguiente: es un error total afirmar que los colores son el último eslabón en una cadena causal de interacción entre propiedades físicas de los objetos y, por ejemplo, el cerebro. Como siempre en filosofía, se puede tanto defender ese punto de vista como rebatirlo, pero especulaciones de esa clase sencillamente no nos conciernen. Lo que es fundamental es entender que no es en relación ni con las propiedades físicas ni en relación con nuestra aparato perceptual que nosotros construimos nuestra plataforma conceptual concerniente a los colores. Nuestros conceptos de colores emergen de nuestros juegos de lenguaje y éstos a su vez se gestan en función de nuestras reacciones y prácticas compartidas (como la clasificación de objetos). Sería sólo como una broma el que alguien pretendiera enseñarle el lenguaje de los colores a un aprendiz de hablante dándole explicaciones de física o de neurofisiología. Nuestro aprendizaje de los significados de los nombres de los colores no tiene absolutamente nada que ver con ellas. Nosotros, los usuarios del lenguaje natural, aprendemos a emplear ese peculiar léxico que es el de los colores en conexión con nuestras reacciones espontáneas y con las series de acciones que desplegamos con miras siempre a obtener algo. El aprendiz normal reacciona de determinada manera frente a ciertos estímulos visuales y lingüísticos y es porque reacciona como lo hacen sus padres, maestros, parientes, nanas y demás que puede pasar a la fase de identificación de colores, esto es, del uso apropiado de los nombres de los colores y a partir de ahí a usar dichos términos como el resto de los usuarios del lenguaje. Es muy importante entender que los seres humanos, esto es, los miembros de la especie lingüística que somos nosotros, aprendemos a coordinar en forma sistemática nuestras reacciones con el uso de las palabras y las reacciones de los demás y que si no coincidiéramos en reacciones el lenguaje sería imposible. El reconocimiento de que esta coordinación es posible y de que es descriptible es importante, porque con ella automáticamente se hace abortar multitud de enredos filosóficos de la más variada índole.

Nuestro punto de vista ayuda a comprender mejor ciertas situaciones que permanecen sistemáticamente sin explicación en los enfoques metafísicos usuales. Es 
bien sabido que el comportamiento de los colores percibidos no sólo no tiene por qué coincidir con su comportamiento físico sino que, de hecho, no lo hace. Desde el punto de vista de la experiencia del color, el blanco es como el amarillo, el negro o el verde, pero el blanco es especial porque desde el punto de vista de la física se comporta de un modo diferente de como lo hacen otros colores. La descripción física del color, sin embargo, no sirve para explicar la experiencia del color, y a la inversa. El físico habla de, por ejemplo, longitudes de onda y el fisiólogo de bastoncitos y conitos, pero ninguna de las propiedades de las que habla la ciencia del color podría servir para describir nuestro conocimiento del color y, por lo tanto, tampoco sirven para explicar nuestros correspondientes juegos de lenguaje. De hecho es perfectamente imaginable que los colores, considerados desde el punto de vista de la física o de la fisiología, tuvieran otras propiedades y que, no obstante, nosotros tuviéramos el mismo aparato conceptual. Las explicaciones a posteriori, como lo son las científicas, por grandiosas que sean, no sirven para explicar lo que es lógicamente previo a ellas.

Un rasgo interesante de nuestro conocimiento de lo que son los colores y sobre el cual Wittgenstein llama la atención es que nosotros nos dejamos impactar por la similitud entre ellos, pero no somos tan duchos en la percepción de sus diferencias. De éstas, como traté de hacer ver, se ocupa la fenomenología pero no, desde luego, mediante la elaboración de una supuesta teoría sino más bien mediante la descripción minuciosa y detallada del comportamiento de los colores en nuestra experiencia visual. En relación con esta tarea, que habremos de dejar de lado en este ensayo, no sé de nadie que haya avanzado más allá de las notas que, en la última etapa de su vida, Wittgenstein nos legara.

RECIBIDO: $27 / 06 / 2019$

RECEIVED: 06/27/2019

APROBADO: $21 / 08 / 2019$

APPROVED: 08/21/2019 